\title{
Possible beneficial association between renin-angiotensin-aldosterone-system blockade usage and graft prognosis in allograft IgA nephropathy: a retrospective cohort study
}

Sehoon Park ${ }^{1,2+}$, Chung Hee Baek ${ }^{3+}$, Heounjeong Go ${ }^{4}$, Young Hoon Kim ${ }^{5}$, Sang-il Min ${ }^{6}$, Jongwon Ha ${ }^{6}$, Yong Chul Kim ${ }^{7}$, Jung Pyo Lee ${ }^{7,8}$, Yon Su Kim ${ }^{1,2,7}$, Kyung Chul Moon ${ }^{9}$, Su-Kil Park ${ }^{3}$ and Hajeong Lee ${ }^{1,7^{*}}$ (D)

\begin{abstract}
Background: Although immunoglobulin A nephropathy (IgAN) is associated with an increased risk of renal allograft failure, evidences for its treatment, including renin-angiotensin-aldosterone system blockade (RAASB) usage, remain limited.

Methods: In this bi-center retrospective cohort study, we included patients who were recently diagnosed with IgAN through allograft biopsies. We identified their 6-month antihypertensive medication prescriptions and investigated the association between the medication types, albuminuria changes, and risk of 5-year death-censored-graft-failure (DCGF). The mixed effect model and cox regression analysis were used.
\end{abstract}

Results: A total of 464 allograft IgAN patients were included: 272, 38, 33, and 121 patients in the no antihypertensive medication, single agent RAASB, single agent beta blocker (BB)/calcium channel blocker (CCB), and combination therapy groups, respectively. High-degree albuminuria after 6 months of allograft IgAN diagnosis was an important prognostic parameter and a partial mediator for the association between the subgroups and 5-year DCGF. The usage of single RAASB was associated with decrement of albuminuria from allograft IgAN diagnosis ( $P$ for interaction $=0.03$ ).

The single BB/CCB group demonstrated significantly worse prognosis than the single RAASB group (adjusted hazard ratio, $2.76[1.09-6.98] ; P=0.03)$.

Conclusions: In conclusion, RAASB may be beneficial for graft prognosis in early allograft IgAN patients who require single antihypertensive medication therapy, by means of reducing albuminuria. Further investigation of treatment strategy in allograft IgAN is warranted.

Keywords: Transplantation, Allograft, IgA nephropathy, Renin-angiotensin-aldosterone system, ACE inhibitors, Angiotensin receptor blockade, Proteinuria

\footnotetext{
* Correspondence: mdhjlee@gmail.com

† Sehoon Park and Chung Hee Baek contributed equally to this work.

${ }^{1}$ Department of Internal Medicine, Seoul National University Hospital, Seoul,

South Korea

${ }^{7}$ Kidney Research Institute, Seoul National University College of Medicine,

Seoul, South Korea

Full list of author information is available at the end of the article
}

(c) The Author(s). 2019 Open Access This article is distributed under the terms of the Creative Commons Attribution 4.0 International License (http://creativecommons.org/licenses/by/4.0/), which permits unrestricted use, distribution, and reproduction in any medium, provided you give appropriate credit to the original author(s) and the source, provide a link to the Creative Commons license, and indicate if changes were made. The Creative Commons Public Domain Dedication waiver (http://creativecommons.org/publicdomain/zero/1.0/) applies to the data made available in this article, unless otherwise stated. 


\section{Background}

Immunoglobulin A nephropathy (IgAN), the most common primary glomerulonephritis during both the pre- and post-transplant eras [1-3], has demonstrated better posttransplant prognosis than other etiologies of end-stage renal disease (ESRD) in the short-term [4]. However, the development or recurrence of IgAN in allograft is associated with an increased risk of graft failure in the long-term $[5,6]$. Although this accelerated graft dysfunction has been repetitively observed $[4,7,8]$, evidence for treatment strategies of allograft IgAN remains limited. As allograft glomerulonephritis is one of the major obstacles in the improvement of long-term graft outcome in modern transplant medicine, the development of an adequate management strategy for allograft IgAN would be beneficial for prolonged graft use.

Renin-angiotensin-aldosterone system blockade (RAASB), which includes angiotensin converting enzyme inhibitors and angiotensin receptor blockades, has been shown to effectively suppress the progression of native IgAN [9-12]. However, the benefits of RAASB during post-transplant periods are still debatable [13-16], although this is mainly related to a low prescription rate of the medications in the post-transplantation period [15]. In addition, whether RAASB usage is associated with better prognosis for primary glomerulonephritis in allograft, including $\operatorname{IgAN}[17,18]$, is uncertain, even though a clinical guideline recommend its usage with a low-grade recommendation level [19].

In this study, we aimed to provide supporting evidence for the possible benefits of RAASB in allograft IgAN. We reviewed our retrospective cohort of allograft IgAN patients and identified their prescribed hypertensive medication categories $[8,20]$. We then compared their prognosis in terms of death-censored-graft-failure (DCGF) and changes in albuminuria levels.

\section{Methods}

\section{Study design and study population}

This was a retrospective cohort study performed in two tertiary referral hospitals in Korea. We included transplant recipients who were diagnosed with pathologically confirmed allograft IgAN. The exclusion criteria were as follows: 1) patients who were diagnosed with allograft IgAN before the year 2000, as electronic medical records did not provide sufficient information related to patients' prescribed medications during this period, and 2) patients with follow-up loss or acute graft failure within 6 months from allograft IgAN diagnosis. The remaining patients were stratified into 4 study groups: 1 ) those who did not receive antihypertensive medications (no medication group); 2) those who received single RAASB-category medications (single RAASB group); 3) those who received single beta-blocker $(\mathrm{BB})$ or calcium channel blocker (CCB) (single BB/CCB group); and 4) those who received multiple categories of medications (combination group). In this studied cohort, no patient received other single agent therapy apart from RAASB, CCB, or $\mathrm{BB}$. A medication history was considered present when an according category was prescribed as a maintenance medication for targeting blood pressure (BP) control within 6 months of allograft IgAN diagnosis.

\section{Allograft IgAN and graft biopsy}

Biopsies to confirm allograft IgAN were mostly performed under the following clinical criteria: a progressive decline in renal function, persistent hematuria, or significant proteinuria of more than $1.0 \mathrm{~g} /$ day $[7,8]$. Some patients underwent protocol biopsies at time-zero and then again 2 weeks post-transplantation. The pathologic diagnosis of allograft IgAN was confirmed when the specimens demonstrated typical immunofluorescence pattern of $\operatorname{IgA} \pm \mathrm{C} 3$ staining in the glomerulus mesangial space.

\section{Data collection}

Patients' clinical characteristics at the time of diagnosis for allograft IgAN were collected, including age, sex, and the relationship between the donor and recipient. Laboratory values, including estimated glomerular filtration rate (eGFR) using the CKD-EPI equation, dipstick albuminuria (none or trace, $1+$, or $\geq 2+$ ), [21] and mean arterial pressure (MAP, $1 / 3$ of systolic BP plus $2 / 3$ of diastolic BP) were collected at the time of biopsy and after 6 months. As RAASB tends to be prescribed to those without progressive decline in graft function in view of its association with acute kidney injury, we calculated the time-averaged eGFRs during the first 3-month-period from allograft IgAN diagnosis, in order to include this variable in our multivariable analysis. The duration from transplantation to allograft IgAN diagnosis was collected, considering that allograft IgAN is a strong time-dependent event [8]. Information on coexisting evidence of acute rejection was also recorded from the pathology reports. Specialized pathologists additionally reviewed the updated Oxford classification, which showed clinical significance also in allograft IgAN [20], in the pathology slides including; M (mesangial hypercellularity), E (endocapillary hypercellularity), S (segmental sclerosis), $\mathrm{T}$ (interstitial fibrosis or tubular atrophy), and $\mathrm{C}$ (cellular or fibrocellular crescent formation). There were missing cases regarding the Oxford classification because some slides were missing and some were at insufficient quality for additional pathology review.

\section{Study outcome}

The main study outcome was 5-year DCGF. The followup period was the duration from allograft IgAN diagnosis until DCGF outcome, follow-up loss, or 5 years. DCGF was defined as a return to maintenance dialysis or re-transplantation, and death events were censored. 


\section{Statistical analysis}

We presented categorical variables as frequencies (percentages) and continuous variables as median values (interquartile ranges). The Kruskal-Wallis test was used to compare continuous variables between the study groups, and the chi-squared tests for the categorical variables. We plotted Kaplan-Meier survival curves to demonstrate survival data, and the log-rank test was used to calculate the $p$-values. The mixed effect model was used to investigate whether usage of a single RAASB had significant interaction with changes in albuminuria or MAP at allograft IgAN diagnosis and after 6 months among those who received antihypertensive medication. We used univariable and multivariable Cox regression analysis to analyze DCGF outcome, and the following variables were included in the multivariable models, if not otherwise specified: age, sex, CKD-EPI eGFR (categorical, $<15, \geq 15$ and 30, $\geq 30$ and $<45, \geq 45$ and $<60, \geq 60$ ), albuminuria (none or trace, $1+$, and $\geq 2+$ ), mean arterial pressure $(\mathrm{mmHg})$, and presence of acute rejection at the time of recurrence. In addition, considering that a difference in longitudinal clinical course might have been present among the studied groups, additional multivariable model analysis was performed, changing the eGFR value at baseline to the time-averaged value within 3 months from allograft IgAN diagnosis. In the multivariable analysis testing the association between the high-degree albuminuria after 6 months and the risk of DCGF in patients without prescribed antihypertensive medications, the eGFR and MAP at 6 months were included in the multivariable model, instead of values at baseline. Whether the presence of high-degree albuminuria was a partial mediator with significance for the association between the antihypertensive medication subgroups and 5-year DCGF was tested using the "mediation" package [22] in R (version 3.4.2, The R Foundation). For the missing values, as they appeared in a random manner, we performed multivariate imputation using classification and the regression trees method with the "mice" package in R [23]. All other statistical analyses were also performed with $\mathrm{R}$. A two-sided $p$ value of less than 0.05 was considered to indicate statistical significance.

\section{Results}

\section{Study population}

Of the 559 allograft IgAN patients, 464 patients were included in the current study after exclusion criteria were applied (Fig. 1). Among them, 100 patients were determined to have initial native IgAN as the cause of ESRD, and 11 had other kidney disease, suggesting possible de novo cases. The other 353 patients had unknown primary etiology or only clinical diagnoses for ESRD. Regarding antihypertensive medication usage, there were $272,38,33$, and 121 allograft IgAN patients in the no medication, single RAASB, single $\mathrm{BB} / \mathrm{CCB}$, and combination groups, respectively.

\section{Characteristics of the study population}

Significant differences in baseline characteristics were found between the study groups (Table 1). Although the age and sex distributions were similar between the groups, the single RAASB group had a significantly longer duration from transplant to allograft IgAN diagnosis. This group had favorable clinical characteristics regarding higher eGFR values and less frequent coexisting acute rejection when they were diagnosed. On the other hand, a substantially higher portion of patients in the single RAASB group had albuminuria and hematuria at baseline. Other medication usage, including tacrolimus prescription ratio among the used calcineurin inhibitors, steroid, or mycophenolic acid, did not differ largely between the studied groups.

\section{Risk factors for 5-year DCGF in the study population}

The risk factors for 5-year DCGF in the study population are shown in Table 2. Male sex, presence of $\mathrm{T}$ in the Oxford classification, and impairment of kidney function, as reflected by decrease in eGFR, were factors prominently associated with an increased risk of DCGF. Particularly, high-degree albuminuria after 6 months of allograft IgAN diagnosis was associated with very high risk for DCGF, which were even comparable to the categorical eGFR variable of $<30$ or $<15 \mathrm{~mL} / \mathrm{min} / 1.73 \mathrm{~m}^{2}$.

Among those who did not require antihypertensive medications, the degree of albuminuria at baseline was not significantly associated with the risk of 5-year DCGF (Additional file 1: Figure S1). However, those with persistent albuminuria or who developed high-degree $(\geq 2+)$ albuminuria after 6 months demonstrated an increased risk of 5-year DCGF. This association was significant even after adjustment for age, sex, time from transplantation to allograft IgAN diagnosis, and eGFR and MAP values after 6 months (adjusted HR, 6.70 [1.51-29.76]; $P=0.01$ ). Meanwhile, the presence of high MAP was not significantly associated with 5-year DCGF in this patient subgroup, suggesting the relative importance of the high-degree albuminuria after 6 months.

By contrast, those who received antihypertensive medications following the diagnosis of allograft IgAN exhibited worse prognosis than those who did not (Additional file 2: Figure S2). Albuminuria and presence of high MAP demonstrated a significant association with 5-year DCGF risk in this patient group; however, baseline high-degree albuminuria lost its significance in multivariable analysis (adjusted HR, 1.31 [0.77-2.23]; $P=0.32$ ). High-degree albuminuria at 6 months remained a significant predictive factor associated with 5-year DCGF in this subgroup (adjusted HR, 2.77 [1.56-4.90]; $P<0.001$ ), also showing the persistent high-degree albuminuria was an important risk factor for DCGF. 


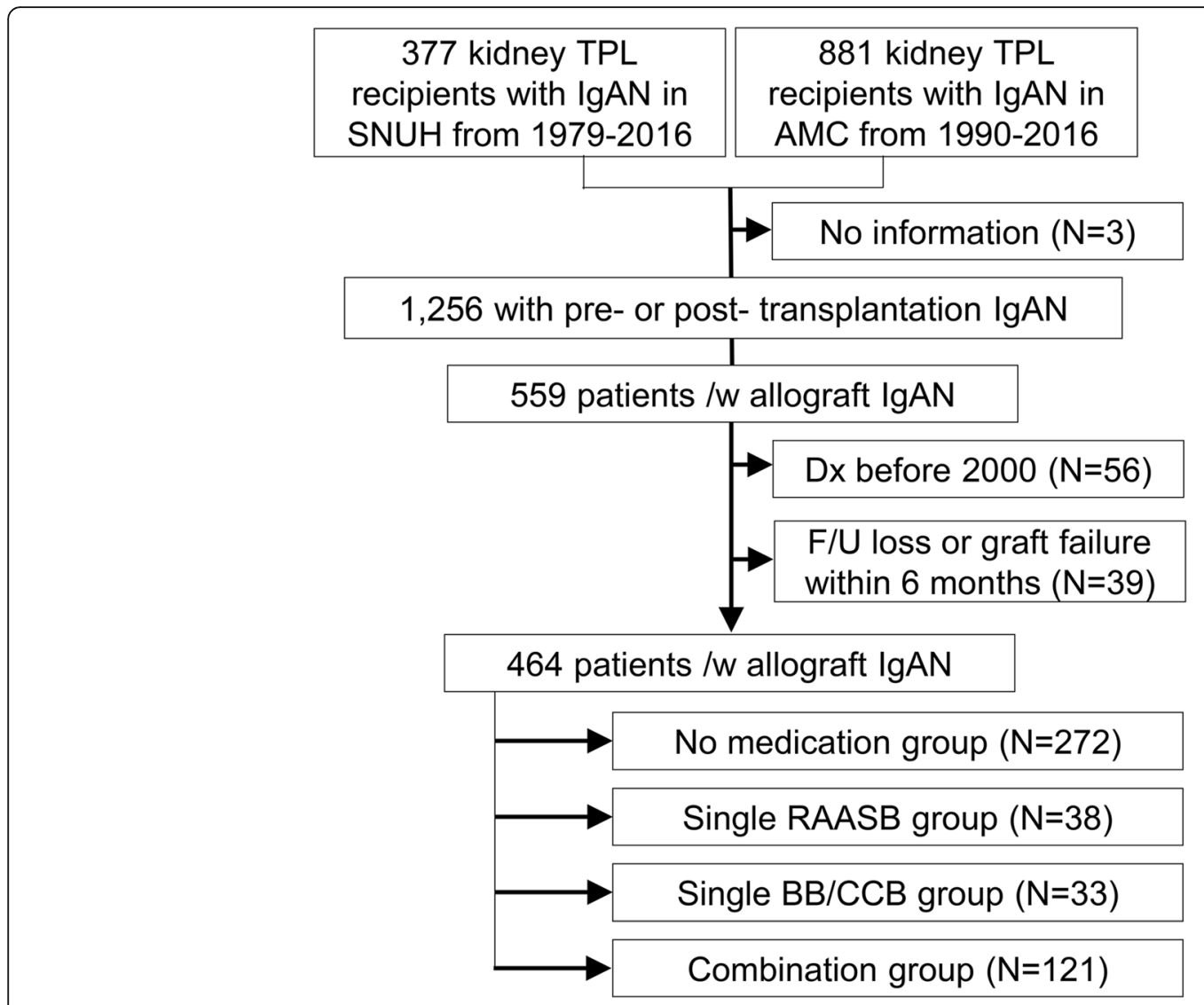

Fig. 1 Study population

Blood pressure and urine dipstick albuminuria according to antihypertensive medications

We analyzed whether differences were present in the urine dipstick results of those with available values both at the time of allograft IgAN diagnosis and after 6 months (Table 3). Compared to the other groups, the single RAASB group had a lower portion of patients with persistent albuminuria or who had developed high-degree albuminuria after 6 months. By contrast, the single BB/ CCB group had a higher portion of allograft IgAN patients who developed high-degree $(\geq 2+)$ dipstick albuminuria at 6 months. Moreover, these differences in changes of albuminuria presence were significant $(\mathrm{P}$ for interaction $=$ 0.03) with single RAASB usage even among those who received single antihypertensive medications, showing that RAASB may be better regarding albuminuria reduction than other drug types.

In terms of blood pressure values, the median MAP was the lowest in the single RAASB group; however, the difference was not statistically significant $(P=0.09)$. In fact, the MAP values became even more similar between the studied groups after 6 months. The changes in high MAP $(>100 \mathrm{mmHg})$ presence $(\mathrm{P}$ for interaction $=0.11$ ) or MAP values ( $\mathrm{P}$ for interaction $=0.28$ ) did not seem to be affected by use of single RAASB. Therefore, we could not identify a significant difference in blood pressure control according to the antihypertensive medication types.

\section{DCGF according to antihypertensive medication categories}

Prognosis for DCGF was significantly different between the studied subgroups (Fig. 2). Patients who did not require antihypertensive medications had the best prognosis, as only 8/272 (2.9\%) patients reached DCGF within 5 years of their allograft IgAN diagnosis. The single RAASB group demonstrated better outcome than the others, with $8 / 38$ (21.1\%) patients developing 5-year DCGF, compared to $15 / 33(45.5 \%)$ and $52 / 121(43.0 \%)$ in the single $\mathrm{BB} / \mathrm{CCB}$ and combination groups, respectively.

The above results were repetitively observed in our regression analysis (Table 4). Compared to the single RAASB group, the no medication group demonstrated better prognosis, while the single $\mathrm{BB} / \mathrm{CCB}$ and combination groups exhibited significantly worse prognoses. Single $\mathrm{BB} / \mathrm{CCB}$ usage and its association with worse DCGF among single agent users remained valid when we included the time-averaged eGFR values. When we 
Table 1 Comparisons of baseline characteristics at the time of allograft IgAN diagnosis according to anti-hypertensive medication prescription

\begin{tabular}{|c|c|c|c|c|}
\hline & $\begin{array}{l}\text { No medication } \\
(N=272)\end{array}$ & $\begin{array}{l}\text { Single RAASB } \\
(N=38)\end{array}$ & $\begin{array}{l}\text { Single } B B / C C B \\
(N=33)\end{array}$ & $\begin{array}{l}\text { Combination } \\
(N=121)\end{array}$ \\
\hline Age at allograft IgAN Dx (years) & $44[34-50]$ & $41[36-47]$ & $44[32-54]$ & $43[35-51]$ \\
\hline Male Sex & $181(66.5 \%)$ & $22(57.9 \%)$ & $25(75.8 \%)$ & $83(68.6 \%)$ \\
\hline $\begin{array}{l}\text { Duration from transplant to allograft } \\
\text { IgAN diagnosis (years) }\end{array}$ & $3.8[0.7-7.5]$ & $6.7[3.2-9.2]$ & $5.4[2.8-8.1]$ & $5.1[$ [2.0- 9.3] \\
\hline \multicolumn{5}{|l|}{ Blood pressure (mmHg) } \\
\hline Systolic BP & 129.0 [117.0-138.0] & 120.0 [110.0-133.0] & 120.0 [115.0-136.0] & $129.0[118.5-139.0]$ \\
\hline Diastolic BP & 78.0 [70.0-86.0] & $74.0[65.0-80.0]$ & $78.0[70.0-85.0]$ & $80.0[70.0-88.0]$ \\
\hline MAP & $94.3[85.3-101.3]$ & 84.8 [79.7-92.7] & 93.0 [85.7-100.7] & $94.3[85.0-101.0]$ \\
\hline \multicolumn{5}{|l|}{ Laboratory values } \\
\hline Serum creatinine (mg/dL) & $1.7[1.4-2.0]$ & $1.5[1.2-2.1]$ & $1.9[1.6-2.6]$ & $1.7[1.4-2.2]$ \\
\hline $\mathrm{eGFR}\left(\mathrm{mL} / \mathrm{min} / 1.73 \mathrm{~m}^{2}\right)$ & $45.0[35.4-57.0]$ & 50.9 [36.9-70.3] & $38.0[27.3-45.9]$ & $45.1[33.8-60.5]$ \\
\hline \multicolumn{5}{|l|}{ Albuminuria } \\
\hline None or trace & $189(69.7 \%)$ & $9(23.7 \%)$ & $20(60.6 \%)$ & $42(35.0 \%)$ \\
\hline $1+$ & $27(10.0 \%)$ & $8(21.1 \%)$ & $7(21.2 \%)$ & $17(14.2 \%)$ \\
\hline$=2+$ & $55(20.3 \%)$ & $21(55.3 \%)$ & $6(18.2 \%)$ & $61(50.8 \%)$ \\
\hline Hematuria & $18(6.6 \%)$ & $18(47.4 \%)$ & $6(18.2 \%)$ & 41 (33.9\%) \\
\hline \multicolumn{5}{|l|}{ Other medication usage } \\
\hline Tacrolimus & $138(50.7)$ & $16(42.1)$ & $20(60.6)$ & $51(42.1)$ \\
\hline Steroid & $257(94.5)$ & $35(92.1)$ & $31(93.9)$ & $112(92.6)$ \\
\hline Mycophenolic acid & $182(66.9)$ & $30(78.9)$ & $24(72.7)$ & $94(77.7)$ \\
\hline \multicolumn{5}{|l|}{ Donor characteristics } \\
\hline Age & 39 [31-47] & 36 [30-42] & 40 [28-47] & $40[30-48]$ \\
\hline Male sex & $151(55.5 \%)$ & $21(55.3 \%)$ & $16(48.5 \%)$ & $61(50.8 \%)$ \\
\hline \multicolumn{5}{|l|}{ Type of donor } \\
\hline Living related & $157(57.7 \%)$ & $24(63.2 \%)$ & $16(48.5 \%)$ & $72(59.5 \%)$ \\
\hline Living unrelated & $60(22.1 \%)$ & $9(23.7 \%)$ & $12(36.4 \%)$ & $23(19.0 \%)$ \\
\hline Deceased & $55(20.2 \%)$ & $5(13.2 \%)$ & $5(15.2 \%)$ & $26(21.5 \%)$ \\
\hline \multicolumn{5}{|l|}{ Pathological findings } \\
\hline Coexisting acute rejection & $94(34.6 \%)$ & $5(13.2 \%)$ & $11(33.3 \%)$ & $33(27.3 \%)$ \\
\hline \multicolumn{5}{|l|}{ Oxford classification } \\
\hline \multicolumn{5}{|l|}{ Mesangial hypercellularity } \\
\hline MO & $142(81.6 \%)$ & $23(79.3 \%)$ & $18(81.8 \%)$ & $56(67.5 \%)$ \\
\hline M1 & $32(18.4 \%)$ & $6(20.7 \%)$ & $4(18.2 \%)$ & $27(32.5 \%)$ \\
\hline \multicolumn{5}{|l|}{ Endocapillary hypercellularity } \\
\hline E0 & $130(78.3 \%)$ & $16(57.1 \%)$ & $16(72.7 \%)$ & $49(59.8 \%)$ \\
\hline E1 & $36(21.7 \%)$ & $12(42.9 \%)$ & $6(27.3 \%)$ & $33(40.2 \%)$ \\
\hline \multicolumn{5}{|l|}{ Segmental sclerosis } \\
\hline so & $108(61.7 \%)$ & $14(48.3 \%)$ & $16(72.7 \%)$ & $43(51.2 \%)$ \\
\hline S1 & $67(38.3 \%)$ & $15(51.7 \%)$ & $6(27.3 \%)$ & $41(48.8 \%)$ \\
\hline \multicolumn{5}{|l|}{ Tubular atrophy/interstitial fibrosis } \\
\hline T0 & $127(72.6 \%)$ & $21(72.4 \%)$ & $14(63.6 \%)$ & $56(67.5 \%)$ \\
\hline $\mathrm{T} 1$ & $33(18.9 \%)$ & $4(13.8 \%)$ & $4(18.2 \%)$ & $17(20.5 \%)$ \\
\hline $\mathrm{T} 2$ & $15(8.6 \%)$ & $4(13.8 \%)$ & $4(18.2 \%)$ & $10(12.0 \%)$ \\
\hline
\end{tabular}


Table 1 Comparisons of baseline characteristics at the time of allograft IgAN diagnosis according to anti-hypertensive medication prescription (Continued)

\begin{tabular}{lllll}
\hline & $\begin{array}{l}\text { No medication } \\
(N=272)\end{array}$ & $\begin{array}{l}\text { Single RAASB } \\
(N=38)\end{array}$ & $\begin{array}{l}\text { Single BB/CCB } \\
(N=33)\end{array}$ & $\begin{array}{l}\text { Combination } \\
(N=121)\end{array}$ \\
\hline Cellular/fibrocellular crescents & & & $29(87.9 \%)$ & $88(72.7 \%)$ \\
C0 & $239(87.9 \%)$ & $30(78.9 \%)$ & $4(12.1 \%)$ & $29(24.0 \%)$ \\
C1 & $30(11.0 \%)$ & $7(18.4 \%)$ & $0(0.0 \%)$ & $4(3.3 \%)$ \\
C2 & $3(1.1 \%)$ & $1(2.6 \%)$ & \\
\hline
\end{tabular}

RAASB Renin-angiotensin-aldosterone system blockades, BB Beta blockers, CCB Calcium channel blockers, IgAN Immunoglobulin A nephropathy, eGFR Estimated glomerular filtration rate, $B P$ Blood pressure, MAP Mean arterial pressure

Table 2 Risk factors for DCGF in the study cohort

\begin{tabular}{lll}
\hline & $\begin{array}{l}{ }^{\mathrm{a}} \text { Adjusted HR } \\
(95 \% \mathrm{Cl})\end{array}$ & $P$ \\
\hline Age (years) & $1.00(0.97-1.02)$ & 0.71 \\
Male sex (.vs female) & $2.50(1.46-4.28)$ & $<$ \\
& & 0.001 \\
Duration from transplant to allograft IgAN & $1.05(0.98-1.12)$ & 0.18 \\
diagnosis (years) & & \\
eGFR (.vs $\left.\geq 60 \mathrm{~mL} / \mathrm{min} / 1.73 \mathrm{~m}^{2}\right)$ & & \\
$\geq 45$ and $<60$ & $1.61(0.68-3.82)$ & 0.28 \\
$\geq 30$ and $<45$ & $2.35(1.02-5.38)$ & 0.04 \\
$\geq 15$ and $<30$ & $6.88(2.94-16.14)$ & $<$ \\
& & 0.001 \\
$<15$ & $7.43(2.28-24.17)$ & $<$ \\
& & 0.001
\end{tabular}

Albuminuria (.vs none or trace)

$\begin{array}{lll}1+\text { at baseline } & 0.95(0.43-2.10) & 0.90 \\ \geq 2+\text { at baseline } & 1.56(0.86-2.83) & 0.15 \\ 1+\text { at } 6 \text { months } & 1.81(0.82-3.98) & 0.14 \\ \geq 2+\text { at } 6 \text { months } & 5.64(3.20-9.93) & < \\ & & 0.001\end{array}$

MAP (mmHg)

High MAP (> $100 \mathrm{mmHg}$ ) at baseline

High MAP (> $100 \mathrm{mmHg}$ ) at 6 months

Coexisting acute rejection

$1.12(0.67-1.90) \quad 0.66$

$1.13(0.72-1.77) \quad 0.59$

$1.07(0.65-1.76) \quad 0.80$

Oxford classification

$\begin{array}{lll}\text { M1 (vs. M0) } & 1.09(0.59-2.03) & 0.78 \\ \text { E1 (vs. E0) } & 1.62(0.89-2.98) & 0.11 \\ \text { S1 (vs. S0) } & 1.36(0.49-3.80) & 0.52 \\ \text { T1 or T2 (vs. T0) } & 2.08(1.21-3.55) & 0.008 \\ \text { C1 or C2 (vs. C0) } & 1.50(0.88-2.54) & 0.13\end{array}$

DCGF Death-censored-graft failure, IgAN Immunoglobulin A nephropathy, eGFR Estimated glomerular filtration rate, MAP Mean arterial pressure

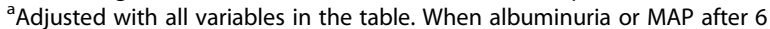
months were included in the model, the values of eGFR, MAP, and the degree of albuminuria at 6 months from diagnosis were included instead the baseline values. Missing values were imputed using the multivariate imputation using classification and the regression trees method divided subgroups according to the presence of confirmed native IgAN, the inferiority for prognosis of single usage $\mathrm{BB} / \mathrm{CCB}$ when compared with prognosis of single RAASB usage was shown only in allograft IgAN with unknown or clinical cause for ESRD (hazard ratio 3.16, 95\% confidence interval 1.17-8.57, $P=0.02)$. On the contrary, in allograft IgAN cases with confirmed native IgAN, the usage of single $\mathrm{BB} / \mathrm{CCB}$ showed non-significant difference regarding prognosis with the single RAASB usage (hazard ratio $2.40,95 \%$ confidence interval $0.44-13.08, P=0.31$ ). In addition, within the combination group, the DCGF prognosis did not significantly differ according to RAASB usage (Additional file 3: Figure S3).

Finally, within the single RAASB group, DCGF was significantly better in those without high-degree albuminuria after 6 months from diagnosis $(P=0.048)$ (Additional file 4: Figure S4). Namely, allograft IgAN patients with high-degree of albuminuria after 6 months from their diagnosis demonstrated poor prognosis, with a 5 -year DCGF rate of $5 / 13$ (38.5\%). By contrast, only 3/21 (14.3\%) single RAASB users progressed to DCGF within 5 years in those without high-degree albuminuria after 6 months. Moreover, in our mediation analysis, high-degree albuminuria after 6 months was a partial mediator of the association between the antihypertensive medication subgroups and DCGF $(P=0.008)$.

\section{Discussion}

In this retrospective study, we investigated the potential benefits of RAASB in allograft IgAN by identifying prescribed hypertensive medication categories and comparing the prognosis in terms of DCGF and changes in albuminuria levels. Our main finding was that allograft IgAN patients who received single RAASB demonstrated better graft outcome than those who received single $\mathrm{BB} /$ $\mathrm{CCB}$ or combination therapy. Among the single agent users, single RAASB usage was associated with a significantly reducing pattern of albuminuria. As the absence of high-degree dipstick albuminuria after 6 months was an important prognostic factor, benefits of RAASB on DCGF might have been related to its protective effect on 
Table 3 Albuminuria and blood pressures at the time of diagnosis and after 6 months of allograft lgAN patients who had complete information

\begin{tabular}{|c|c|c|c|c|c|c|}
\hline \multirow[b]{2}{*}{ Dipstick albuminuria } & \multicolumn{3}{|c|}{ At allograft IgAN diagnosis } & \multicolumn{3}{|l|}{ After 6 months } \\
\hline & None or trace & $1+$ & $\geq 2+$ & None or trace & $1+$ & $\geq 2+$ \\
\hline No medication $(N=216)$ & $143(66.2 \%)$ & $22(10.2 \%)$ & $51(23.6 \%)$ & $166(76.9 \%)$ & $26(12.0 \%)$ & $24(11.1 \%)$ \\
\hline Single RAASB $(N=38)$ & $9(23.7 \%)$ & $8(21.1 \%)$ & $21(55.3 \%)$ & $18(47.4 \%)$ & $7(18.4 \%)$ & $13(34.2 \%)$ \\
\hline Single $B B / C C B(N=28)$ & 19 (67.9\%) & $5(17.9 \%)$ & $4(14.3 \%)$ & $15(53.6 \%)$ & $4(14.3 \%)$ & $9(32.1 \%)$ \\
\hline Combination $(N=94)$ & $34(36.2 \%)$ & $10(10.6 \%)$ & $50(53.2 \%)$ & $33(35.1 \%)$ & $18(19.1 \%)$ & $43(45.7 \%)$ \\
\hline Blood pressure & Systolic BP & Diastolic BP & MAP & Systolic BP & Diastolic BP & MAP \\
\hline No medication $(N=268)$ & 129.0 [117.0-138.0] & $78.0[70.0-85.5]$ & $95.0[85.5-102.7]$ & 125.0 [116.0-135.0] & 76.0 [68.0-84.0] & $92.7[84.5-100.2]$ \\
\hline Single RAASB $(N=33)$ & 120.0 [110.0-133.0] & 74.0 [65.0-80.0] & $88.7[81.7-98.7]$ & 122.0 [118.0-136.0] & 81.0 [72.0-88.0] & 94.7 [90.0-103.3] \\
\hline Single $B B / C C B(N=28)$ & 120.0 [114.5-136.0] & $79.0[69.5-87.0]$ & $93.2[84.5-100.3]$ & 126.5 [116.5-140.0] & $79.0[70.5-84.0]$ & $94.2[85.8-103.3]$ \\
\hline Combination $(N=104)$ & $129.0[118.5-139.5]$ & 80.0 [70.0-88.0] & $96.3[88.0-103.3]$ & $128.0[116.0-140.0]$ & $79.0[70.0-87.0]$ & 93.7 [85.0-103.8] \\
\hline
\end{tabular}

IgAN Immunoglobulin A nephropathy, RAASB Renin-angiotensin-aldosterone blockades, BB Beta blockers, CCB Calcium channel blockers, BP Blood pressure, MAP Mean arterial pressure

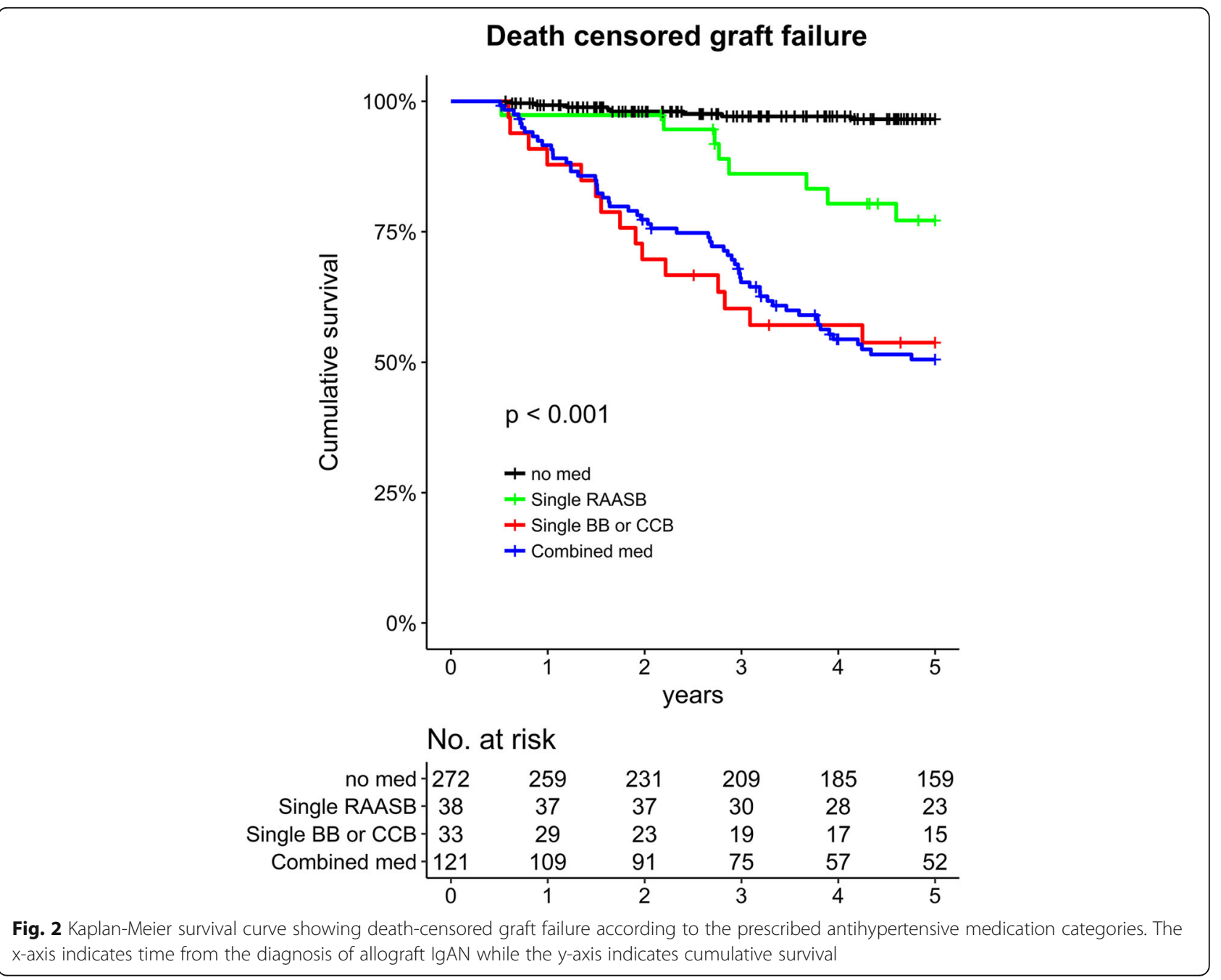


Table 4 Use of HTN medication within 6 months from allograft IgAN Dx and its' association with the risk of DCGF

\begin{tabular}{|c|c|c|c|c|c|c|}
\hline & Univariable & & Multivariable - model & & Multivariable - model & \\
\hline & HR $(95 \% \mathrm{Cl})$ & $P$ & Adjusted HR (95\% Cl) & P & Adjusted HR (95\% Cl) & $P$ \\
\hline Single RAASB & Ref. & & Ref. & & Ref. & \\
\hline No medication & $0.15(0.06-0.40)$ & $<0.001$ & $0.17(0.06-0.48)$ & 0.001 & $0.17(0.06-0.49)$ & 0.001 \\
\hline Single BB/CCB & $2.74(1.16-6.47)$ & 0.02 & 3.05 (1.19-7.79) & 0.02 & $2.88(1.13-7.37)$ & 0.03 \\
\hline Combination & $2.76(1.32-5.79)$ & 0.007 & $2.72(1.24-5.96)$ & 0.01 & $2.28(1.04-4.99)$ & 0.04 \\
\hline
\end{tabular}

HR Hazard ratio, CI Confidence interval, RAASB Renin-angiotensin-aldosterone system blockades, BB Beta blockers, CCB Calcium channel blockers

${ }^{a}$ Model 1. Adjusted for clinicopathologic characteristics at the time of allograft IgAN; age, sex, CKD-EPI eGFR (categorical, $<15, \geq 15$ and $30, \geq 30$ and $<45, \geq 45$ and $<60, \geq 60$ ), albuminuria (none or trace, $1+$, and $\geq 2+$ ), mean arterial pressure $(\mathrm{mmHg})$, pathologic components of the Oxford classification $(\mathrm{M}, \mathrm{E}, \mathrm{S}, \mathrm{T}$, and $\mathrm{C}$ components), presence of acute rejection at the time of recurrence

${ }^{\mathrm{b}}$ Model 2. The adjustment variable of eGFR value at allograft IgAN diagnosis in above Model 1 was substituted for time-averaged eGFR within 3 months after diagnosis of allograft IgAN

urine albumin (a surrogate marker of urine protein) loss. However, the result was only significant in the allograft IgAN cases without confirmed native IgAN but was not evident in the recurred IgAN cases in that IgAN was also identified in native kidneys.

RAASB has been widely used for many renal diseases because of its protective effect on the cardiovascular and renal systems. The benefits of RAASB on renal prognosis were well-demonstrated within proteinuric primary glomerulonephritis or diabetic nephropathy patients $[10,11,24,25]$. There is a possibility that the medication may also be beneficial for graft outcome after kidney transplantation; however, this has yet to be confirmed [13-16]. In addition, while clinical guidelines for kidney transplantation recipients suggest prescribing RAASB for those with recurrent glomerulonephritis and proteinuria [19], the recommendation level remains low. Our study showed possible benefits of RAASB usage as the initial single agent for better graft prognosis in allograft IgAN, which was not clearly indicated in a previous report [18]. In addition to the preceding study that showed RAASB's protective effect on proteinuria among allograft IgAN patients [17], our study also demonstrated that the benefits may lead to better graft survival.

As stated, this potential benefit might be related to the identified medications' protective effect on dipstick albuminuria, which is a surrogate marker of proteinuria [26, 27]. The reno-protective effect of RAASB is mainly derived from reduced intraglomerular pressure and proteinuria, which was considered to be independent of the underlying disease [17]. In the present study, high-degree albuminuria after certain period from the diagnosis of allograft IgAN was one of the most important predictors of DCGF, while MAP or baseline albuminuria demonstrated only weaker associations. This suggests that in a state of allograft IgAN, the severity of glomerulonephritis, which may be reflected by persistent albuminuria, may be an important parameter or damaging factor for worse graft prognosis. Considering the close linkage between single RAASB usage, decreased proportion of patients with high-degree albuminuria, and better
DCGF, albuminuria reduction might be the moderator of the association between RAASB and better graft outcome among single agent users. In addition, this may also be supported by the fact failure to reduce albuminuria during the first 6 months from allograft IgAN diagnosis, even within the single RAASB group, was associated with worse allograft outcome. Clinicians should keep this result in mind and consider RAASB when single-agent antihypertensive therapy is required for allograft IgAN patients; however, they should also consider the side effects with caution [14, 28].

Still, it should be noted that the possible benefits of single agent RAASB over single $\mathrm{BB}$ or $\mathrm{CCB}$ was not confirmed herein for the recurred IgAN cases with confirmed native IgAN. In our previous study, the clinical significance of the Oxford classification was different according to allograft IgAN subtypes [20]. In allograft IgAN with confirmed native IgAN cases, $\mathrm{M}, \mathrm{E}, \mathrm{S}$, and C components, which may reflect intraglomerular disease activity, were important prognostic parameters, but in the cases without confirmed native IgAN, only the $\mathrm{T}$ was a significant pathologic risk factor. When relating this with the current study results, appropriate immunosuppression may be more important in the "recurred IgAN" cases, which showed more rapid progression and in which pathologic parameters related to intraglomerular disease activity showed prognostic importance. On the other hand, in allograft IgAN cases without confirmed native IgAN, which were possibly de novo ones in which progressed tubular injury, the $\mathrm{T}$, was an important prognostic factor, reduction of proteinuria to decrease progressive allograft injury might have been more important than in recurred IgAN cases. Our study suggests a future study investigating whether actual pathophysiology of recurred IgAN and de novo IgAN is different. Moreover, studying whether usage of immunosuppression is associated with difference in prognosis of recurred IgAN cases, particularly with high-risk pathologic parameters, may help developing individualized treatment strategy for allograft IgAN.

Patients who did not require antihypertensive medication following allograft IgAN diagnosis demonstrated 
better prognosis than those who did. By contrast, those who received combination therapy displayed poorer prognosis, regardless of the prescription of RAASB. A required medication dosage or the presence of uncontrolled BP might have been the more important parameter related to graft prognosis than the albuminuria in these patients. Furthermore, this implies that the benefit of RAASB usage in advanced allograft IgAN requiring multiple antihypertensive agents remains unclear. Therefore, the current study only supports the usage of RAASB when singleagent therapy is required.

Several unresolved limitations should be considered in our study. These are mainly related to the difficulty of conducting a study concerning post-transplant medication usage, especially for non-rejection allograft diseases. First, a hidden confounding effect might be present. It is true that clinicians may hesitate to prescribe RAASB in patients with rapidly declining graft function. Although we studied the long-term outcomes and attempted to adjust the potential confounders, this innate difference in antihypertensive medication indications might have affected our results. Second, as many patients were receiving combination therapy, a comparison between the single agents, which would give the most important consequence, was performed only in a limited number of patients. However, as this was one of the largest cohorts of allograft IgAN to date, this might be the only reported evidence that may demonstrate the possible benefits of RAASB usage on graft survival in allograft IgAN. Third, due to the study's retrospective nature, whether the addition of RAASB in allograft IgAN patients is necessary could not be directly answered herein. In addition, several patients were already on RAASB, and a sufficient wash-out period could not have been secured. While a prospective study may be necessary to solve this problem, the inclusion of sufficient number of trial subjects may be an issue. Lastly, we used dipstick albuminuria values, a surrogate marker of proteinuria [21], and amounts of proteinuria were not measured using the 24-h collection or random urine protein/ creatinine ratio. Although this is a significant limitation, the use of the variable was necessitated by the limited number of allograft IgAN patients with available quantified proteinuria results.

\section{Conclusions}

The potential benefit of RAASB on allograft IgAN prognosis was highlighted in the present study. Clinicians may consider using RAASB for allograft IgAN cases without confirmed native IgAN, or de novo cases, particularly in those at an early stage who do not require combination therapy. Additional studies regarding the treatment strategies for primary glomerulonephritis in renal transplant recipients are warranted.

\section{Additional files}

Additional file 1: Figure S1. Presence of high-degree albuminuria ( $\geq$ $2+$ ) or high MAP (> $100 \mathrm{mmHg}$ ) and their association with 5-year DCGF within patients those did not receive any antihypertensive agents. (PDF $138 \mathrm{~kb}$ )

Additional file 2: Figure S2. Presence of high-degree albuminuria ( $\geq$ $2+$ ) or high MAP (> $100 \mathrm{mmHg}$ ) and their association with 5-year DCGF within patients those did not receive any antihypertensive agents. (PDF $185 \mathrm{~kb}$ )

Additional file 3: Figure S3. 5-year DCGF according to usage of RAASB within the combination group. (PDF $106 \mathrm{~kb}$ )

Additional file 4: Figure S4. 5-year DCGF according to presence of a high-degree $(\geq 2+)$ albuminuria at baseline or after 6 months from allograft IgAN diagnosis within the single RAASB group. (PDF $101 \mathrm{~kb}$ )

\begin{abstract}
Abbreviations
AMC: Asan medical center; BB: Beta blockers; BP: Blood pressure; CCB: Calcium channel blockers; DCGF: Death-censored graft failure; eGFR: Estimated glomerular filtration rate; ESRD: End stage renal disease; IgAN: Immunoglobulin A nephropathy; MAP: Mean arterial pressure; RAASB: Renin-angiotensin-aldosterone-system blockades; sCr: Serum creatinine; SNUH: Seoul national university hospital
\end{abstract}

\section{Acknowledgements}

Not applicable.

\section{Authors' contributions}

$\mathrm{HL}$ is the principal investigator and gave the research idea. SP, CHB, SKP, and $\mathrm{HL}$ planned the initial cohort collection. SP, CHB, YCK, HG, YHK, JPL, SM, JH, KCM collected the raw data. YSK, SKP, HL reviewed the study protocol. SP, $\mathrm{CHB}$, and $\mathrm{HL}$ performed the statistical analysis. $\mathrm{HG}$ and $\mathrm{KCM}$ provided support in pathological aspects and performed pathological review. All authors participated in interpreting the results and critically contributed to manuscript drafting. All authors contributed the manuscript finalization and approved the final manuscript for submission.12.

\section{Funding}

This study was supported by a grant from the Ministry of Health and Welfare (No. HI15C2632), a grant from the National Research Foundation of Korea (No. 2019R1A2C2011465), and a grant from the Ministry of Science, ICT and Future Planning of Korea (No. 2015M3C9A2054342). The funder played no role in performing the study; the study was performed independently by the authors.

\section{Availability of data and materials}

The datasets used and/or analyzed during the current study are available from the corresponding author on reasonable request.

\section{Ethics approval and consent to participate}

The study was approved by the institutional review board (IRB) of Seoul National University Hospital (SNUH, IRB No: H-1701-008-819) and Asan Medical Center (AMC, IRB No: S2017-0369-0001). We performed this study in accordance with the principles of the Declaration of Helsinki. Informed consent was waived under approval by the attending IRB because of the retrospective nature of the study. The clinical and research activities being reported are consistent with the Principles of the Declaration of Istanbul as outlined in the 'Declaration of Istanbul on Organ Trafficking and Transplant Tourism'."

\section{Consent for publication}

Not applicable.

\section{Competing interests}

The authors declare that they have no competing interests.

\section{Author details}

${ }^{1}$ Department of Internal Medicine, Seoul National University Hospital, Seoul, South Korea. ${ }^{2}$ Department of Biomedical Sciences, Seoul National University 
College of Medicine, Seoul, South Korea. ${ }^{3}$ Department of Internal Medicine, Asan Medical Center, University of Ulsan College of Medicine, Seoul, South Korea. ${ }^{4}$ Department of Pathology, Asan Medical Center, University of Ulsan College of Medicine, Seoul, South Korea. ${ }^{5}$ Department of Surgery, Asan Medical Center, University of Ulsan College of Medicine, Seoul, South Korea. ${ }^{6}$ Department of Surgery, Seoul National University Hospital, Seoul National University College of Medicine, Seoul, South Korea. ${ }^{7}$ Kidney Research Institute, Seoul National University College of Medicine, Seoul, South Korea. ${ }^{8}$ Department of Internal Medicine, Seoul National University Boramae Medical Center, Seoul, South Korea. ${ }^{9}$ Department of Pathology, Seoul National University Hospital, Seoul, South Korea.

Received: 9 June 2019 Accepted: 27 August 2019

Published online: 11 September 2019

\section{References}

1. Nair R, Walker PD. Is IgA nephropathy the commonest primary glomerulopathy among young adults in the USA? Kidney Int. 2006;69(8):1455-8.

2. Lee H, Kim DK, Oh KH, Joo KW, Kim YS, Chae DW, Kim S, Chin HJ. Mortality and renal outcome of primary glomerulonephritis in Korea: observation in 1,943 biopsied cases. Am J Nephrol. 2013;37(1):74-83.

3. Abbas F, El Kossi M, Jin JK, Sharma A, Halawa A. Recurrence of primary glomerulonephritis: review of the current evidence. World J Transplant. 2017;7(6):301-16.

4. Wyld ML, Chadban SJ. Recurrent IgA nephropathy after kidney transplantation. Transplantation. 2016;100(9):1827-32.

5. Briganti EM, Russ GR, McNeil JJ, Atkins RC, Chadban SJ. Risk of renal allograft loss from recurrent glomerulonephritis. N Engl J Med. 2002;347(2):103-9.

6. Allen PJ, Chadban SJ, Craig JC, Lim WH, Allen RDM, Clayton PA, TeixeiraPinto A, Wong G. Recurrent glomerulonephritis after kidney transplantation: risk factors and allograft outcomes. Kidney Int. 2017;92(2):461-9.

7. Han SS, Huh W, Park SK, Ahn C, Han JS, Kim S, Kim YS. Impact of recurrent disease and chronic allograft nephropathy on the long-term allograft outcome in patients with IgA nephropathy. Transpl Int. 2010;23(2):169-75.

8. Park S, Baek CH, Cho H, Yu MY, Kim YC, Go H, Kim YH, Lee JP, Min Sl, Ha J, et al. Glomerular crescents are associated with worse graft outcome in allograft IgA nephropathy. Am J Transplant. 2019;19(1):145-55.

9. Coppo R, Peruzzi L, Amore A, Piccoli A, Cochat P, Stone R, Kirschstein M, Linne T. IgACE: a placebo-controlled, randomized trial of angiotensin-converting enzyme inhibitors in children and young people with IgA nephropathy and moderate proteinuria. J Am Soc Nephrol. 2007;18(6):1880-8.

10. Praga M, Gutierrez E, Gonzalez E, Morales E, Hernandez E. Treatment of IgA nephropathy with ACE inhibitors: a randomized and controlled trial. J Am Soc Nephrol. 2003;14(6):1578-83.

11. Maschio G, Cagnoli L, Claroni F, Fusaroli M, Rugiu C, Sanna G, Sasdelli M, Zuccala A, Zucchelli P. ACE inhibition reduces proteinuria in normotensive patients with IgA nephropathy: a multicentre, randomized, placebocontrolled study. Nephrol Dial Transplant. 1994;9(3):265-9.

12. KDIGO work group. KDIGO 2012 clinical practice guideline for the evaluation and management of chronic kidney disease. Kidney Int Suppl. 2013;3:1):1-150.

13. Hiremath S, Fergusson DA, Fergusson N, Bennett A, Knoll GA. Reninangiotensin system blockade and long-term clinical outcomes in kidney transplant recipients: a meta-analysis of randomized controlled trials. Am J Kidney Dis. 2017:69(1):78-86.

14. Cruzado JM, Rico J, Grinyo JM. The renin angiotensin system blockade in kidney transplantation: pros and cons. Transpl Int. 2008;21(4):304-13.

15. Opelz G, Zeier M, Laux G, Morath C, Dohler B. No improvement of patient or graft survival in transplant recipients treated with angiotensin-converting enzyme inhibitors or angiotensin II type 1 receptor blockers: a collaborative transplant study report. J Am Soc Nephrol. 2006;17(11):3257-62.

16. Hiremath S, Fergusson D, Doucette $S$, Mulay AV, Knoll GA. Renin angiotensin system blockade in kidney transplantation: a systematic review of the evidence. Am J Transplant. 2007;7(10):2350-60.

17. Oka K, Imai E, Moriyama T, Akagi Y, Ando A, Hori M, Okuyama A, Toki K, Kyo M, Kokado Y, et al. A clinicopathological study of IgA nephropathy in renal transplant recipients: beneficial effect of angiotensin-converting enzyme inhibitor. Nephrol Dial Transplant. 2000;15(5):689-95.

18. Courtney AE, McNamee PT, Nelson WE, Maxwell AP. Does angiotensin blockade influence graft outcome in renal transplant recipients with IgA nephropathy? Nephrol Dial Transplant. 2006;21 (12):3550-4.
19. Kasiske BL, Zeier MG, Chapman JR, Craig JC, Ekberg H, Garvey CA, Green MD, Jha V, Josephson MA, Kiberd BA, et al. KDIGO clinical practice guideline for the care of kidney transplant recipients: a summary. Kidney Int. 2010; 77(4):299-311.

20. Park S, Go H, Baek CH, Kim YH, Kim YC, Yang SH, Lee JP, Min SI, Ha J, Song EY, et al. Clinical importance of the updated Oxford classification in allograft IgA nephropathy. Am J Transplant. 2019; Epub ahead of print.

21. Constantiner M, Sehgal AR, Humbert L, Constantiner D, Arce L, Sedor JR, Schelling JR. A dipstick protein and specific gravity algorithm accurately predicts pathological proteinuria. Am J Kidney Dis. 2005;45(5):833-41.

22. Dustin Tingley TY, Hirose K, Keele L, Imai K. Mediation: R package for causal mediation analysis. J Stat Softw. 2014;59(5):1-38.

23. Buuren SVG-OK. Mice: multivariate imputation by chained equations in R. J Stat Softw. 2011:45:1-67.

24. Lewis EJ, Hunsicker LG, Bain RP, Rohde RD. The effect of angiotensinconverting-enzyme inhibition on diabetic nephropathy. The collaborative study group. N Engl J Med. 1993;329(20):1456-62.

25. The GISEN Group (Gruppo Italiano di Studi Epidemiologici in Nefrologia). Randomised placebo-controlled trial of effect of ramipril on decline in glomerular filtration rate and risk of terminal renal failure in proteinuric, non-diabetic nephropathy. Lancet. 1997;349(9069):1857-63.

26. Amer H, Fidler ME, Myslak M, Morales P, Kremers WK, Larson TS, Stegall MD, Cosio FG. Proteinuria after kidney transplantation, relationship to allograft histology and survival. Am J Transplant. 2007;7(12):2748-56.

27. Kimata N, Tanabe K, Ishikawa N, Ozu H, Sonda K, Yumura W, Toma H, Nihei $\mathrm{H}$, Ota K, Takahashi K. Correlation between proteinuria and prognosis of transplant IgA nephropathy. Transplant Proc. 1996;28(3):1537-9.

28. Morrone LF, Di Paolo S, Logoluso F, Schena A, Stallone G, Giorgino F, Schena FP. Interference of angiotensin-converting enzyme inhibitors on erythropoiesis in kidney transplant recipients: role of growth factors and cytokines. Transplantation. 1997;64(6):913-8.

\section{Publisher's Note}

Springer Nature remains neutral with regard to jurisdictional claims in published maps and institutional affiliations.
Ready to submit your research? Choose BMC and benefit from:

- fast, convenient online submission

- thorough peer review by experienced researchers in your field

- rapid publication on acceptance

- support for research data, including large and complex data types

- gold Open Access which fosters wider collaboration and increased citations

- maximum visibility for your research: over $100 \mathrm{M}$ website views per year

At BMC, research is always in progress.

Learn more biomedcentral.com/submissions 